ausführten. Die Möglichkeit, zwischen diesen sich widersprechenden Rechnungen durch direkte Wärmemessung zu entscheiden, sah Jacobsohn, ,wegen des zu langsamen Ablaufs der Reaktion" nicht gegeben.

Um einen weiteren Beitrag zur Theorie der fermentativen Katalyse zu gewinnen, haben wir nun sowohl die Wärmebildung in der Reaktion Fumarsäure $\rightarrow$ Apfelsäure als auch den Wärmeverbrauch in der Reaktion Apfelsäure $\rightarrow$ Fumarsäure calorimetrisch gemessen und -3800 cal bzw. +3900 cal erhalten.

Gewinnung des Ferments. Kalhsleber wurde durch die Latapiemuihle getrieben, der Brei mit einem halben Volumen Wasser angerüht, 24 h bei $0^{0}$ aufbewahrt und zentrifugiert. Die überstehende Lösung stand vor Beginn der Versuche 10 Tage unter Toluol im Kühlschrank.

Ansatz: $16 \mathrm{ccm}$ Substratlösung: $4 \mathrm{ccm}$ Veronalpuffer; 0,15 cem Ferment; im (iasraum Stickstoff: $p_{\mathrm{H}} 6,8 ; \vartheta 24,3^{0}$. In dem Versuch $\perp$ ist das Substrat, in den Versuchen $\mathbf{F}$ und $\mathrm{G}$ der Puffer fortgelassen.

Analyse. Bestimmung der Apfelsäure polari- metrisch nach Jacobsoh $n^{5}$, der Fumarsäure gravimetrisch nach $A$. Hahn u. W. Ha a rmann ${ }^{8}$.

Ergebnisse. Versuch A: Das Einkippen des Ferments in Pufferlösung verläuft praktisch thermoneutral. Versuch B: Mischt man Malat und Fumarat nach Maßgabe der Gleichgewichtskonstante und setzt Ferment zu, so tritt kein Vorgang ein, dessen Wärmetönung die Fehlergrenze der Messung ïberschritte. Unter dieser Voraussetzung konnten wir für Hin- und Rückreaktion entgegengesetzt gleiche Wärmemengen finden (Versuche C...G) (Tab.5).

Für die Theorie folgt, daß die Gleichung der Reaktions-Isochore durch die Werte für Gleichgewichtskonstante und Wärmetönung in den Grenzen der Methoden befriedigt wird. Das Ferment ist also ohne Einfluß auf die thermodynamisch geforderte Gleichgewichtslage.

Ich danke Frl. G. Sch ü z für ilıre IIilfe bei der Ausführung der Messungen.

\& Z. Biol. 87, 107 [1928].

\title{
Auslösung von Polyploidie durch Kälte bei Drosophila melanogaster
}

\author{
Von Hans Bauer \\ Aus dem Kaiser-Wilhelm-Institut für Biologie, Hechingen \\ (Z. Naturforschg. 1, 35-38 [1946]; eingegangen am 1. September 1945)
}

Durch Kältehehandlung von 2 Stdn. alten Embryonen wird eine Polyploiditierung von Urkeimzellen erzielt, die am Auftreten triploider Nachkommen fe-tgestellt wird.

$\mathrm{B}$ ei Drosophila melanogasier sind triploide \& fund tetraploide Fliegen beider Geschlechter bekannt. Erstere werden seit langem regelmäßig gezüchtet; 4 -Tiere aber sind nur vereinzelt beobachtet worden ${ }^{1}$. Für die Klärung mancher genetischer Fragen besonders auch der Mutationsforschung wären konstante tetraploide Stämme nützlich. Sie müßten sich erhalten lassen, wenn bei ihrem Aufbau attached-X-Chromosomen verwandt würden ${ }^{2}$. Voraussetzung für die willkürliche Herstellung solcher 4n-Stämme ist es, sich von dem Aufsuchen spontan auf-

1 C. B. Bridges, Cytological and genetic basis of sex. Sex a. intern. secretions. 2nd ed. 15-63 [1939].

2 H. B a u e r, Fortschr. d. Zool. N.F. 7, 256 [1943]. tretender Polyploider unabhängig zu machen, also ihre experimentelle Auslösung zu erreichen. Diesem Ziel dienten die im folgenden beschriebenen Versuche.

In der botanischen Genetik herrscht heute zur experimentellen Polyploidisierung das Colchicin-Verfahren vor. Seine Anwendung auf Tiere ist durch die Schwierigkeit, das Colchi(rin in einer für den Organismus unschädlichen Weise wirksam an die Keimzellen heranzubringen, noch stark eingeschränkt. Es liegen nur ganz vereinzelte positive Versuchsberichte vor. Um nicht erst mühsam einen geeigneten Weg zur Uberwindung der Schwierigkeiten suchen zu müssen, wurde auf das älteste Verfahren zurückgegriffen, die von J. J. Gerassi- 
mo $w^{3}$ eingeführte Kältebehandlung. Es wurden sich entwickelnde Eier der Kälte ausgesetzt; sie reagieren bei ihrer geringen Dicke gleichmäßiger, als von den Larven angenommen werden kann, und enthalten Urkeimzellen, die noch eine längere Mitosenfolge zu durchlaufen haben. Aus einer polyploid gewordenen Urkeimzelle muß, wenn sie nicht entwicklungsbenachteiligt ist, eine größrere Menge polyploider Keimzellen entstehen, daher ist eine gruppenweise Häufung von polyploiden Nachkommen zu erwarten, die zugleich ein wichtiger Anhaltspunkt für den Erfolg des Versuches ist. Es wurden daher frühe Embryonalstadien, bei denen die Urkeimzellen als Polzellen ausgetreten sind, behandelt. Das ist bei 2 Stdn. alten Eiern der Fall ${ }^{4}$.

Als Kriterium für die gelungene Polyploidisierung der Urkeimzellen wurde das Auftreten triploider Töchter bei der Kreuzung von (im Embryonalstadium) kältebehandelten $\&+\mathrm{mit}$ unbehandelten $\sigma^{x} \sigma^{x}$ benutzt. Die $\& \&$ hatten die Konstitution Notch ${ }^{8} / y e l l o w$ white $\left(N^{8} / y w\right)$, die $\sigma^{7} \sigma^{7}$ facet-notchoid ( $\left.f a^{n o}\right)$. Der Faktor $f a^{n o}$ wurde deshalb gewählt, weil er in Kombination mit Notch larvenletal ist ${ }^{5}$. Dadurch wird die Anzahl der auszuzählenden $\& \&$ auf die Hälfte reduziert und es tritt normalerweise bei ihnen ebenso wie bei den $\sigma^{x} \sigma^{x}$ (infolge Absterbens der $N^{8} / Y$-Zygoten) nur eine Klasse auf. Außerdem sollte durch $f a^{n o}$ in Anwesenheit von $N^{3}$ eine Klasse der möglichen triploiden $\mathrm{F}_{1-}$ \& $\&$ gekennzeichnet werden. Die durch die Kältebehandlung erzielten tetraploiden Oogonien haben die Konstitution $N^{8 / N^{8} / y w / y w}$. Bei Zufallsverteilung der X-Chromosomen in den Reifeteilungen entstehen also 3 Sorten von diploiden Eizellen: $N^{s / N^{8}}, N^{8} / y w$ und $y w / y w$ im Verhältnis $1: 4: 1$, demnach 3 Sorten von triploiden Zygoten: $N^{8} / N^{*} / f a^{n o}, N^{*} / y w / f a^{n o}$ und $y w / y w / f a^{n o}$. Die ersteren sind wie die diploiden $N^{s} / f a^{n o}-\mathrm{Zy}$ goten letal, die aus den letzteren hervorgehenden $f q$ unterscheiden sich nicht von $y w / f a^{n o_{-}}$ $f+$. Die $N^{8} / y w / f a^{n o}+q$ dagegen sollten nach der von B a u e r ${ }^{5}$ gegebenen Deutung den NotchPhänotypus aufweisen. Nach dieser Deutung

3 Bull. Soc. Imp. Natur. Moscou No. 1 u. 2. 185 [1901]

${ }_{4}^{4}$ D. F. P o u ls on, Act. scient. et ind. (Paris) 498, 1 [19:37].

${ }^{5}$ H. B a u er, Z. Vererbgsi. 81, 374 [1943]. stellt facet-notchoid ein Deficiency eines der Elemente einer im normalen Genotypus der Art rorhandenen Genduplikation dar; bei NotchFaktoren ist dagegen das ganze Gendublett entfernt. Für die normale Flügelausbildung sind je $\mathrm{X}$-Chromosom durchschnittlich mindestens 1,5 dieser Gene notwendig. Der Notch-Phänotypus kommt bei einem Gen-X-Verhältnis von 1,0 zustande $\left(N^{*} /+;\left\lceil a^{n o} /\left\lceil a^{n o} ;\left\lceil a^{n o}-\sigma^{7}\right)\right.\right.\right.$. Ein Verhältnis $<1$ ist letal. Bei den zu erwartenden $3 n-q+$ der Konstitution $N^{s} / y w / a^{n o}$ liegt ein Verhältnis 1,0 vor $(0+2+1: 3)$; sie sollten also Notch-Flügel besitzen. Das hat sich in den Versuchen durch Weiterzucht der Triploiden bestätigt, womit die vorgeschlagene Deutung der Natur des fano-Faktors eine weitere Stütze erhält.

Daß auch die diploiden $y w / f^{n o-} Q 9$ in einem geringen Prozentsatz kleine Flügelausschnitte aufweisen ${ }^{5}$, stört nicht, da sie an der fehlenden Aderverdickung stets von den $3 \mathrm{n}$ - 9 q unterschieden werden können.

Durch die Auszählung der Notch-Fliegen werden also zwei Drittel der triploiden $\&-\mathrm{Zy}_{\mathrm{y}}$ goten erfaßt. Von den diploiden $\uparrow$-Zygoten ist dagegen nur die Hälfte lebensfähig. Das Verhältnis von $3 n-: 2 n-Z y g o t e n$ ist also etwas kleiner als das der $3 n-: 2 n-q$ \&

Der Versuchsgang war der folgende: Unbefruchtete $N^{8} /+-0 Q$ wurden bei $25^{0}$ mit $y w$ - $\hat{\jmath} \hat{s}$ in Röhrchen gebracht, die in üblicher Weise mit agarbedeckten Objektträgern beschickt waren. Auf der Höhe der Legeperiode wurden die Objektträger in einstündigen Intervallen gewechselt. Die abgelegten Eier wurden dann nach Erreichen eines Durchschnittsalters von 2 Stdn. mit den Agarplättchen unmittelbar auf Eis oder auf eine Eiskochsalzmischung gebracht, so daß sie Temperaturen von $0^{0} \mathrm{bzw}$. $-4^{0}$ ausgesetzt waren. Nach $1 / 2-4$ Stdn. wurden die Eier in Kulturflaschen gebracht, wo sie bei $25^{\circ}$ die weitere Entwicklung durchmachten. Die schlüpfenden $N^{s} / y w-q q$ wurden unbefruchtet abgesammelt und zu 2 bis 4 zusammen mit $\left\{a^{n o-\hat{\jmath}} \hat{\jmath}\right.$ in Flaschen angesetzt.

Über die Behandlungsdauer in den Einzelversuchen gibt Tab. 1 Auskunft, in der zugleich die Daten über die durch die Behandlung bedingte Sterblichkeit zusammengefaßt sind. Von jeder Temperatur und Behandlungsdauer wurden 3-4 Einzelversuche gemacht, die in der Tabelle nur gesammelt angeführt sind. Die Sterblichkeitswerte in diesen Teilversuchen streuen stark. Im ganzen tritt mit zunehmender 


\begin{tabular}{|c|c|c|c|c|c|}
\hline $\begin{array}{c}\text { Tem- } \\
\text { peratur }\end{array}$ & $\begin{array}{c}\text { Behand- } \\
\text { lungs- } \\
\text { dauer } \\
\text { in } \\
\text { Stunden }\end{array}$ & $\begin{array}{l}\text { Anzahl } \\
\text { der } \\
\text { behan- } \\
\text { delten } \\
\text { Eier }\end{array}$ & $\begin{array}{c}\text { Anzahl } \\
\text { der } \\
\text { erwar- } \\
\text { teten } \\
\text { Fliegen }\end{array}$ & $\begin{array}{l}\text { Anzahl } \\
\text { der ge- } \\
\text { schlipf- } \\
\text { ten } \\
\text { Fliegen }\end{array}$ & $\begin{array}{c}\text { kälte- } \\
\text { bewirkte } \\
\text { Mor- } \\
\text { talität } \\
\text { in } \%\end{array}$ \\
\hline \multirow{4}{*}{$0^{0}$} & 1 & 603 & 301 & 144 & 52,2 \\
\hline & 2 & 82.5 & 412 & 244 & 40,8 \\
\hline & 3 & 1290 & 645 & 165 & 74,4 \\
\hline & 4 & 757 & 378 & 119 & 68,5 \\
\hline \multirow{4}{*}{$-4^{0}$} & $1 / 2$ & 1226 & 613 & 315 & 48,6 \\
\hline & 1 & 779 & 389 & 224 & 42,4 \\
\hline & $1 \%$ & 966 & 483 & 108 & 77,6 \\
\hline & 2 & 921 & 460 & 158 & 65,7 \\
\hline
\end{tabular}

Tab. 1. Sterblichkeit der Fliegen auf frühen Entwicklungsstadien nach Kältebehandlung.

Behandlungsdauer und abnehmender Temperatur aber eine Zunahme der Sterblichkeit auf. Es ist sehr wahrscheinlich, daß die Sterblichkeit nicht ausschlaggebend auf einer Partialpolyploidisierung des Embryos beruht; denn diese dürfte in erster Linie durch den Abkühlungsschock bedingt und unabhängig sein von der weiteren Dauer der Kälteeinwirkung uiber eine wohl nur kurze Minimalzeit hinaus.

Die Ergebnisse der Nachkommenzuchten der kialtebehandelten $f f$ faßt Tab. 2 zusammen. Aus ihr geht hervor, daß der Prozentsatz triploider $q$ \& in den Kontrollzuchten auferordentlich gering ist; er beträgt nur $0.015 \%$. Die 3 Triploiden traten einzeln in verschiedenen Kulturen auf. Anders ist das Bild in den Versuchs-

\begin{tabular}{|c|c|c|c|c|c|}
\hline \multirow{2}{*}{\multicolumn{2}{|c|}{ Versuchsart }} & \multicolumn{3}{|c|}{ geschlüpfte $F_{1}$-Fliegen } & \multirow{2}{*}{$\begin{array}{l}3 n-\% \text { in } \\
\% \text { aller }+\%\end{array}$} \\
\hline & & $0^{\circ}$ & $2 n-7$ & $3 n-6$ & \\
\hline \multicolumn{2}{|c|}{ Kontrolle } & 19226 & 20513 & 3 & 0,015 \\
\hline Temp. & Stunden & & & & \\
\hline \multirow{4}{*}{$0^{0}$} & 1 & 397 & 516 & 2 & \\
\hline & 2 & 3706 & 4197 & 21 & \\
\hline & 3 & 1572 & 1683 & - & \\
\hline & 4 & 269 & 459 & 3 & \\
\hline Gesamt & $\cdots$ & 5944 & 6855 & 26 & 0,38 \\
\hline \multirow{4}{*}{$-4^{0}$} & $1 / 2$ & 702 & 673 & 4 & \\
\hline & 1 & 619 & 644 & 13 & \\
\hline & $11 / 2$ & 342 & 366 & 1 & \\
\hline & 2 & 669 & 817 & 1 & \\
\hline Gesamt & $\ldots$ & $2: 332$ & 2500 & 19 & 0,75 \\
\hline
\end{tabular}

Tab. 2. Auftreten triploider $q 9$ nach Kältebehandlung der Muitter im Embryonalzustand.

zuchten: Bei $0^{0}$-Behandlung wurden $0,38 \%$, bei $-4^{0}$-Behandlung $0,75 \%$ triploide $\&$ f erhalten. Die Steigerung gegenüber den Kontrollen beläuft sich, wenn auch eine genaue statistische Bewertung nicht möglich ist, auf das 25-bis 50 -fache. Das ist zwar immer noch wenig, reicht

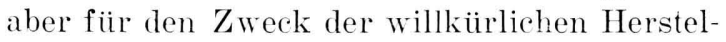
lung polyploider Stämme voll aus. Wieweit reale Unterschiede zwischen den Versuchen mit verschiedener Temperatur und Behandlungsdauer vorliegen, lält sich im einzelnen nicht sagen.

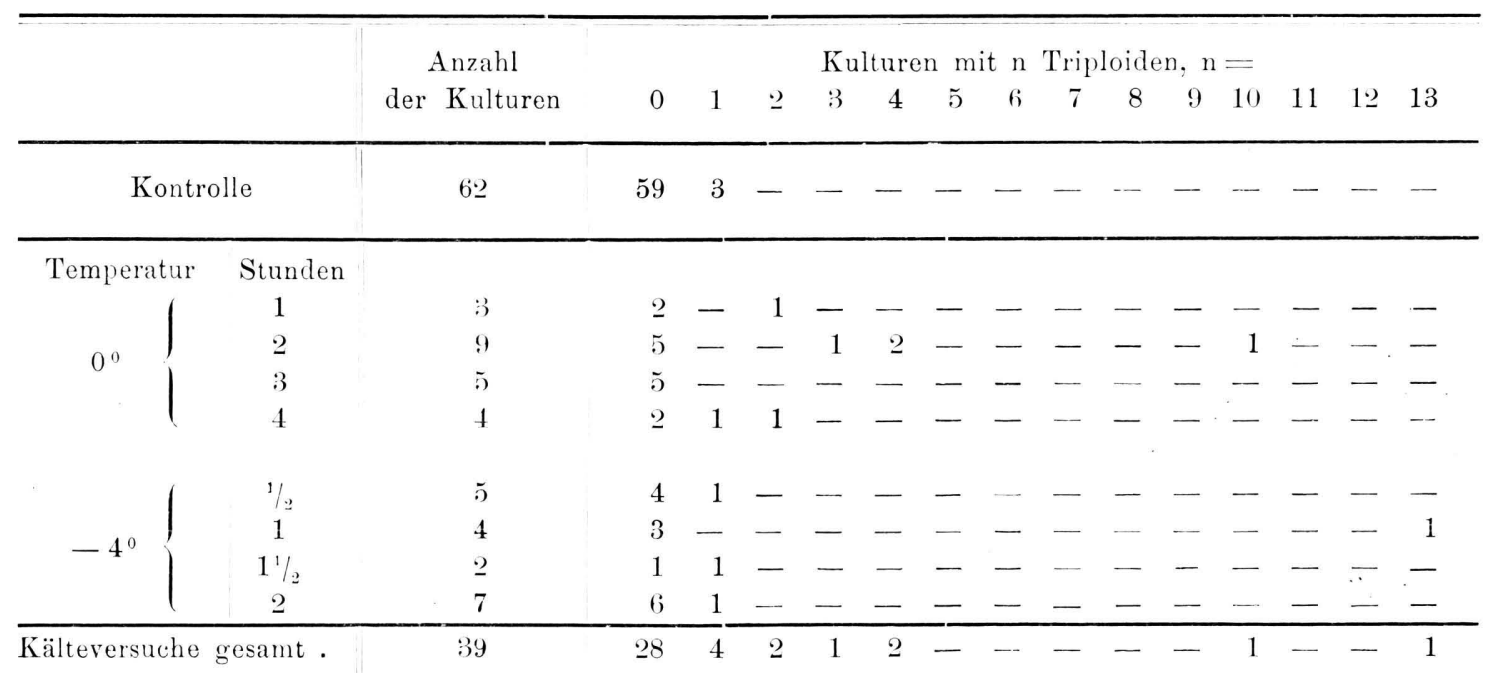

Tab. 3 Verteilung der Triploiden auf die Einzelzurhten. 
Besonders auffällig ist die Verteilung der Triploiden auf die einzelnen Kulturen (Tab. 3). Ohne weiteren statistischen Vergleich ist es klar, daß solche Verteilungen, wie sie besonders bei $0^{0} 2$ Stdn. und bei $-4^{0} 1$ Std., aber auch bei den anderen Versuchsgruppen mit mehr als einem $3 n-q$ beobachtet wurden, nicht eine Zufallsverteilung wiedergeben ${ }^{6}$. Sie zeigen vielmehr eine Häufung der Triploiden in den Einzelkulturen an. Die Erklärung dafür ist nach dem oben Gesagten offensichtlich; während das Auftreten der Triploiden in den Kontrollversuchen wohl allein auf meiotische Störungen zurückgeht, müssen einzelne der behandelten $f q$ als Folge der Polyploidisierung der Urkeimzellen tetraploide Ovarteile, einzelne

6 Unter der vereinfachenden Annahme, daß die Einzelkulturen gleiche Individuenanzahlen aufwiesen, berechnet sich die Wahrscheinlichkeit dafiir, daß die Gesamtverteilung der Triploiden in den Kälteversuchskulturen (Tab. 3, unterste Zeile) einer $\mathrm{P}$ o i s s on-Verteilung entspricht, a uf $\mathrm{P}<10-6$. oder Gruppen von Eiröhren, besitzen, aus denen mehrere diploide Eizellen entstehen.

Daß nur so wenige größere Gruppen von Triploiden beobachtet wurden, liegt zum Teil daran, daß nur ${ }^{1 / 3}$ der diploiden Eizellen erfaßt werden; denn nicht nur ${ }^{1 / 3}$ der $3 n-q+$ entzieht sich der Beobachtung oder stirbt ab, sondern auch eine den 3n-Zygoten gleiche Zahl von XXY-Zygoten bleibt unberïcksichtigt. Außerdem werden auch wohl nicht wenige hypo- oder hyperdiploide Eizellen entstehen. Die Beschränkung der Triploidenzeugung auf nur vereinzelte $f f$ schließlich liegt zweifellos daran, daß nur in einem geringen Teil der behandelten Eier einzelne Urkeimzellen gerade in dem für die Restitutions kernbildung geeigneten Mitosestadium sind. Es ist wahrscheinlich, daß eine mehrfache Kältebehandlung mit kurzen Erwärmungspausen einen noch erheblich günstigeren Versuchsausfall gibt.

Hrn. G. W i e r t z, der die Versuche ausgeführt hat, bin ich für seine Hilfe dankbar.

\title{
Unregelmäßige Chromosomenverteilung durch eine Mutation bei Ephestia kühniella Z.
}

\author{
Von Alfred Kühn und Dore-Li Woywod \\ Aus dem Kaiser-Wilhelm-Institut für Biologie, Hechingen \\ (Z. Naturforschg. 1, 38-44 [1946]; eingegangen am 1. September 1945)
}

\begin{abstract}
Die unvollständig dominante Mutation Mo bewirkt unregelmäßige Chromosomenverteilung, meist in einer der ersten embryonalen Teilungen. In dem reinen Stamm entstehen etwa 20\% Mosaiktiere, meist Halbseitenmosaiks, die verschieden helle Flügelschuppen haben. Im Mittel ist die eine Seite gegenüber der Flügelhelligkeit der gleichseitigen Tiere des Stammes aufgehellt, die andere verdunkelt. Nach Einkreuzung spalten verschiedene rezessive mutierte Allele nicht gekoppelter Gene mosaikhaft heraus. Mo bewirkt also die unregelmäßige Verteilung verschiedener Chromosomen. Das auf der einen Seite ausfallende Chromosom kann ganz verloren gehen oder der anderen Seite zugeteilt werden. Auch zweifach monosome Flügel können entstehen.
\end{abstract}

$\mathrm{I}_{\mathrm{u}}^{\mathrm{n}}$ n unseren Zuchten von Ephestia kühniella und von Ptychopoda seriata kommen ab und zu Mosaiktiere vor. Drei Fälle wurden früher schon beschrieben: von Ephestia ein Sektorialmosaik $^{1}$ und ein Halbseitenmosaik ${ }^{2}$, in

1 A. K ühn, Versuche üher die Wirkungsweise der Erbanlagen. Naturwiss. 24, 6 | 1936].

2 A. K ühn, Zur Entwicklungsphysiologie der Schmetterlingsschuppen. Biol. Zhl. 61, 136 ff. [1941]. denen bei einem $+\mid b$-Heterozygoten eine Seite $b$ zeigte, und von Plychopoda ein Halbseitenmosaik ${ }^{3}$, bei dem in einer wildfarbigen Zucht auf einer Seite die der Mutation At entsprechende Flïgelbeschuppung erschien.

${ }^{3}$ A. K ü h n u. M. v. Eng elh a r d t, Uber zwei melanistische Mutationen (At und $n i$ ) von Ptychopoda seriata Schrk. Biol. Zhl. 63, 264 ff. [1943]. 